\title{
Fission cross-sections, prompt fission neutron and $\gamma$-ray emission in request for nuclear applications
}

\author{
F.-J. Hambsch ${ }^{1}$, P. Salvador-Castiñeira ${ }^{1,2}$, S. Oberstedt ${ }^{1}$, A. Göök ${ }^{1}$, R. Billnert ${ }^{1}$ \\ ${ }^{1}$ EC-JRC-IRMM, Retieseweg 111, B-2440 Geel, Belgium \\ ${ }^{2}$ National Physical Laboratory, Hampton Road, Teddington, Middlesex, UK, TW11 OLW
}

\begin{abstract}
In recent years JRC-IRMM has been investigating fission cross-sections of ${ }^{240,242} \mathrm{Pu}$ in the fast-neutron energy range relevant for innovative reactor systems and requested in the High Priority Request List (HPRL) of the OECD/Nuclear Energy Agency (NEA). In addition to that, prompt neutron multiplicities are being investigated for the major isotopes ${ }^{235} \mathrm{U},{ }^{239} \mathrm{Pu}$ in the neutron-resonance region using a newly developed scintillation detector array (SCINTIA) and an innovative modification of the Frisch-grid ionisation chamber for fission-fragment detection. These data are highly relevant for improved neutron data evaluation and requested by the OECD/Working Party on Evaluation Cooperation (WPEC). Thirdly, also prompt fission $\gamma$-ray emission is investigated using highly efficient lanthanide-halide detectors with superior timing resolution. Again, those data are requested in the HPRL for major actinides to solve open questions on an under-prediction of decay heat in nuclear reactors. The information on prompt fission neutron and $\gamma$-ray emission is crucial for benchmarking nuclear models to study the de-excitation process of neutron-rich fission fragments. Information on $\gamma$-ray emission probabilities is also useful in decommissioning exercises on damaged nuclear power plants like Fukushima Daiichi to which JRC-IRMM is contributing. The results on the ${ }^{240,242} \mathrm{Pu}$ fission cross section, ${ }^{235} \mathrm{U}$ prompt neutron multiplicity in the resonance region and correlations with fission fragments and prompt $\gamma$-ray emission for several isotopes will be presented and put into perspective.
\end{abstract}

\section{Introduction}

Data needs for advanced reactor systems and improved nuclear data evaluations have recently been the focus of research at JRC-IRMM. Here mainly the High Priority Request List (HPRL) of the OECD/Nuclear Energy Agency (NEA) is consulted as well as recommendations given in reports of the Working Party on International Evaluation Co-operation (WPEC) of the OECD/NEA. This has resulted in investigations of fission cross sections of the highest priority targets for fast reactors, ${ }^{240,242} \mathrm{Pu}(\mathrm{n}, \mathrm{f})[1]$. The neutron scintillation detector array SCINTIA has been developed to investigate prompt fission neutron emission in resonance neutron induced fission, based on requests from WPEC related investigations $[2,3]$. And finally, measurements of $\gamma$-ray emission in several actinides have been performed and are planned in the near future in request of the HPRL entries H:3 and H:4 [4]. In the following sections results of the three items mentioned will be highlighted. 


\section{Fission cross sections}

For the next generation of nuclear power plants (GEN-IV), four of the six designs currently under study are based on a fast neutron-energy spectrum instead of a thermal one. Consequently, there are upcoming needs for the nuclear data community to meet more stringent requirements, in order to improve the accuracy on the performance of simulations codes in this energy region. Addressing these aspects, a sensitivity study was performed by the Nuclear Energy Agency (NEA) [1] with the outcome of a list of high priorities of the most important isotopes and their relevant quantities. Not only for isotopes that eventually would be part of the fuel mixture, but, also, for structural materials intended to surround the reactor core. Within the high priority list it is requested to improve the accuracy of the neutron induced fission cross section of ${ }^{240,242} \mathrm{Pu}$ from the current $5 \%, 20 \%$ to a target value of $1-3 \%$, $5 \%$, respectively.

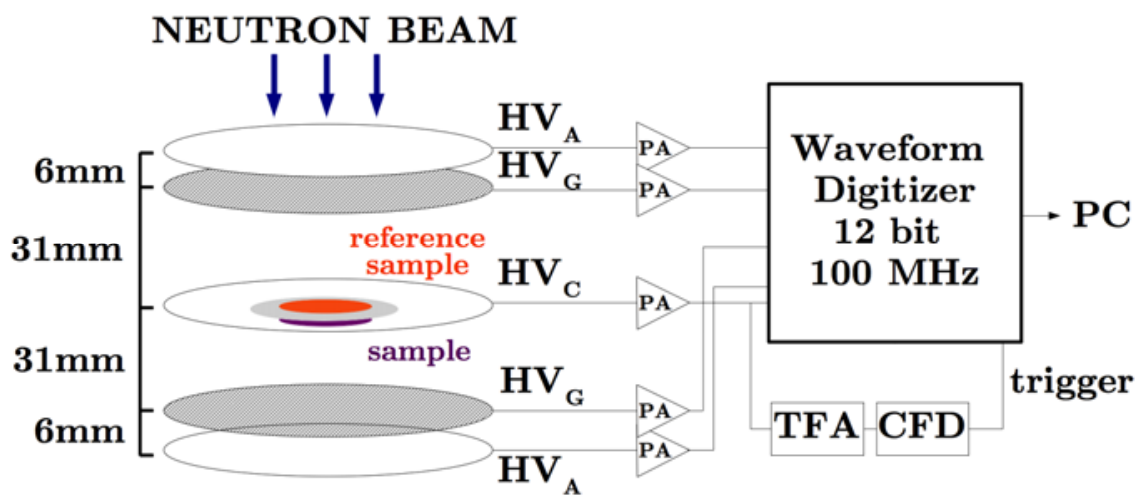

Figure 1. Scheme of the experimental set-up.

\subsection{Experimental setup}

The respective experiments have been performed at the JRC-IRMM Van de Graaff accelerator during the past years. The main difference to previous works in literature was that three reference targets have been used, namely ${ }^{235,238} \mathrm{U}$ and ${ }^{237} \mathrm{~Np} .{ }^{235} \mathrm{U}(\mathrm{n}, \mathrm{f})$ is a primary reference standard, ${ }^{238} \mathrm{U}(\mathrm{n}, \mathrm{f})$ a secondary standard above $2 \mathrm{MeV}$ and ${ }^{237} \mathrm{~Np}(\mathrm{n}, \mathrm{f})$ is no standard, but has a lower threshold and higher fission cross section than ${ }^{238} \mathrm{U}(\mathrm{n}, \mathrm{f})$. A double Frisch grid ionisation chamber was used in back to back geometry for fission fragment detection. The signals of the chamber were digitised using Waveform digitizers and the full traces of the fission fragments were stored for off-line analysis.

This is the second innovation compared to previous literature works. A schematic scheme of the experimental set-up is given in Fig. 1. Digital signal processing is used to deduce the fission crosssection values. More details are given in Refs. [5,6]. The incident neutron energy range covered was from $0.3 \mathrm{MeV}$ to $3 \mathrm{MeV}$. The used targets were prepared at the JRC-IRMM sample preparation group. Since the samples are the most important ingredients to this experiment, a lot of effort has been invested to verify the homogeneity and total mass [7]. Also the spontaneous fission half-lives of ${ }^{240,242} \mathrm{Pu}$ have been re-measured with unprecedented statistical accuracy [8].

\subsection{Experimental results}

In Figs. 2 and 3 the resulting fission cross section values are given using different colours for the different reference targets. In addition the most recent evaluations are also given. It is obvious that for ${ }^{240} \mathrm{Pu}(\mathrm{n}, \mathrm{f})$ our results reproduce the fission threshold very well but in the plateau region the difference to the evaluations is about $5 \%$. For ${ }^{242} \mathrm{Pu}(\mathrm{n}, \mathrm{f})$ our results show a deviation already in the threshold region where the present data tend to a less steep rise of the fission cross section. Furthermore the 
resonance like structure around $1.1 \mathrm{MeV}$ seen in the evaluations is not present in our data. Furthermore in the plateau region up to about $2.5 \mathrm{MeV}$ our data are $7-9 \%$ lower than the evaluations.

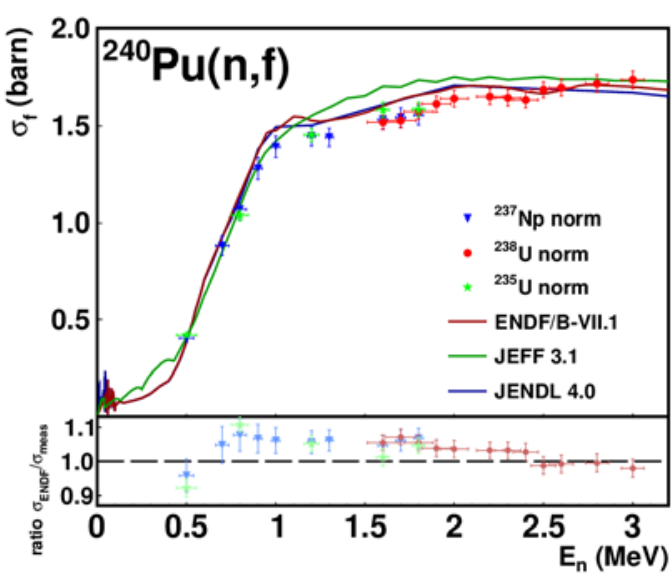

Figure 2. Fission cross section results for ${ }^{240} \mathrm{Pu}(\mathrm{n}, \mathrm{f})$ using three different reference samples as given in the legend compared to the most recent evaluations.

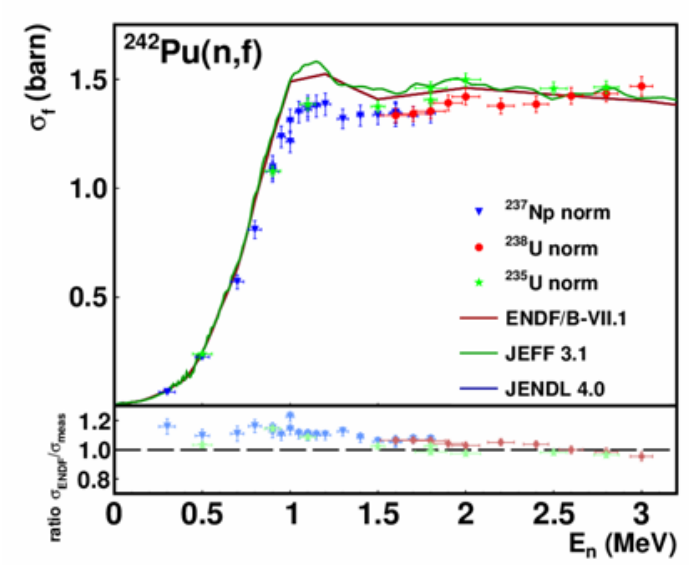

Figure 3. Fission cross section results for ${ }^{240} \mathrm{Pu}(\mathrm{n}, \mathrm{f})$ using three different reference samples as given in the legend compared to the most recent evaluations.

Figs. 4 and 5 bring the present results into perspective to selected previous literature results. The weighted average of the present fission cross section results for ${ }^{240,}{ }^{242} \mathrm{Pu}(\mathrm{n}, \mathrm{f})$ are shown using three different reference samples as given in the legend and compared to literature data and the most recent evaluations.

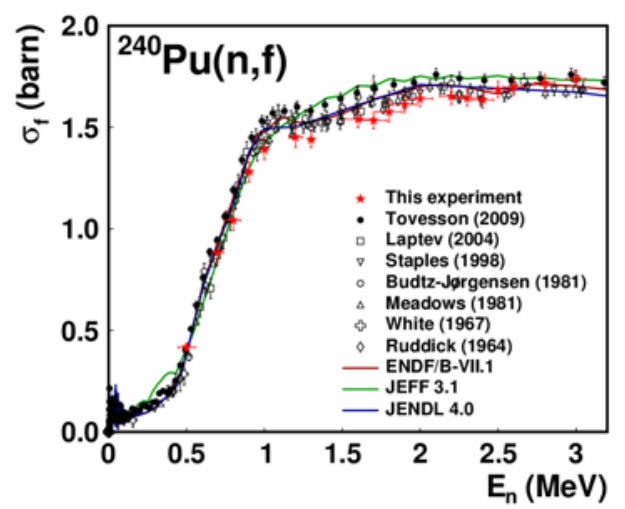

Figure 4. Weighted average of the present fission cross section results for ${ }^{240} \mathrm{Pu}(\mathrm{n}, \mathrm{f})$ using three different reference samples as given in the legend compared to literature data and the most recent evaluations.

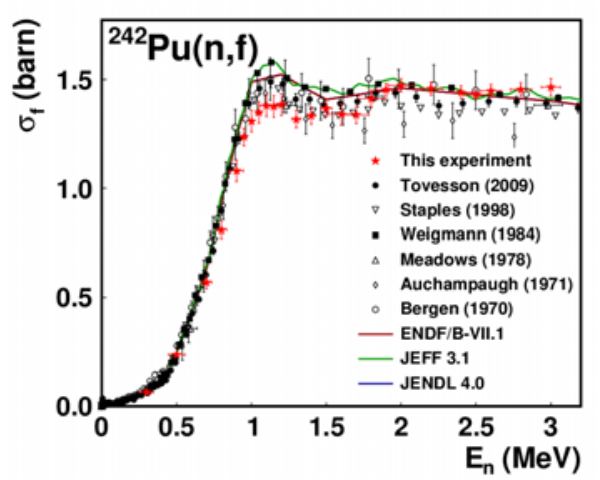

Figure 5. Weighted average of the present fission cross section results for ${ }^{242} \mathrm{Pu}(\mathrm{n}, \mathrm{f})$ using three different reference samples as given in the legend compared to literature data and the most recent evaluations.

\section{Prompt neutron emission in fission}

In understanding the fission process knowledge of prompt fission neutron emission is of particular importance. Information about the properties of prompt fission neutrons, their multiplicities and energy distributions could give answers to questions related not only to the neutron emission itself, but also relevant to the formation of the fission fragments, the sharing of excitation energy among them and the time scale of the process. To improve nuclear modelling and similarly the evaluation of 
nuclear data the knowledge about fluctuations in the prompt neutron multiplicity as a function of incident neutron energy is requested for the major actinides ${ }^{235} \mathrm{U}$ and ${ }^{239} \mathrm{Pu}[2,3]$. Fluctuations in fission fragment mass and total kinetic energy (TKE) for both isotopes have been observed in resonance-neutron induced fission [9, 10]. Independently, the fluctuation in the number of emitted neutrons has been observed, too [11]. In view of the fact that both neutron number and fission fragment properties have been found to vary it is necessary to study the correlations of prompt neutron multiplicity and fission fragments properties in the resonance region [12]. In the most severe cases the relative impact was as large as $20-30 \%$. Hence an experimental campaign to investigate correlations of prompt fission neutron multiplicity with fragment properties in resonance neutron induced fission on ${ }^{235} \mathrm{U}$ has been started at JRC-IRMM.

\subsection{Experimental setup}

The present experimental setup for investigating correlations of prompt neutrons with fission fragments in resonance neutron induced fission on ${ }^{235} \mathrm{U}$ is illustrated in Figs. 6 and 7. The fission target is placed inside the ionization chamber at a distance of about $9.2 \mathrm{~m}$ from the neutron production target of the GELINA facility. An array of neutron detectors (SCINTIA) is employed to improve the fission-neutron coincidence count rate. The SCINTIA array consists of 7 NE213 equivalent liquid scintillators (Scionix LS-301) and 5 para-therphenyl detectors, a photo is shown in Fig. 6. In this setup with an array of neutron detectors, each detector forms an axis of symmetry around which the orientation of the fission axis needs to be known for the purpose of transforming the emission properties into the fission fragment rest frame. Hence the traditional ionization chamber has been improved with a position sensitive readout structure, which allows determination of all three space coordinates of the fission fragment's direction of travel. For data acquisition a 22 channel fully digital system is used. The digital technique has shown many advantages over the analogue technique for fission fragment spectroscopy with ionization chambers (see e.g. Ref. [13]).

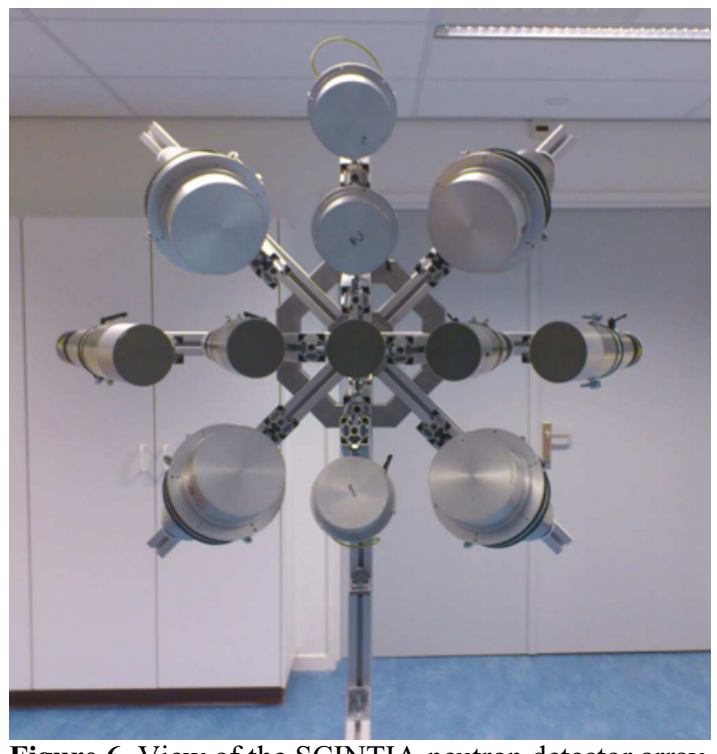

Figure 6. View of the SCINTIA neutron detector array in the laboratory.

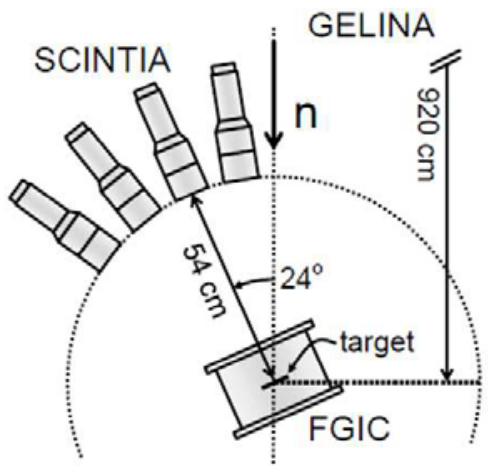

Figure 7. Schematic view of the experimental setup at the GELINA flight path station.

The acquisition is triggered by the current signal from the ionization chamber cathode, giving the instant of a fission event in time with a resolution better than $1 \mathrm{~ns}$ FWHM. For each fission event the digital waveforms of all channels, sampled at $400 \mathrm{MS} / \mathrm{s}$ with 14-bit resolution, are stored on disk for further off-line processing. The incident neutron time-of- flight is derived from the timestamp of each 
event. The system has been validated using the spontaneous fission of ${ }^{252} \mathrm{Cf}$. More detail is given in Refs. $[14,15]$.

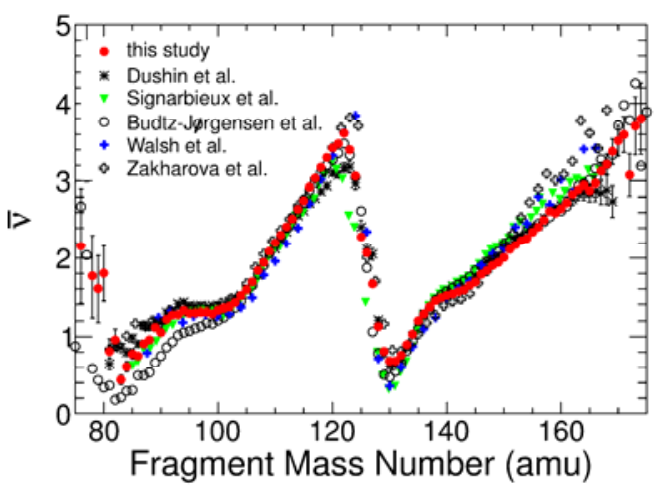

Figure 8 Average total prompt fission neutron multiplicity as a function of the pre-neutron fragment mass number, in comparison with experimental data from literature.

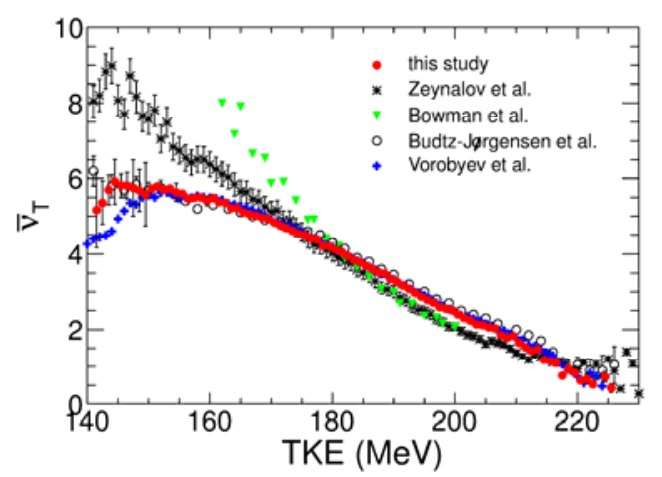

Figure 9. Average total prompt fission neutron multiplicity as a function of TKE, in comparison with experimental data from literature.
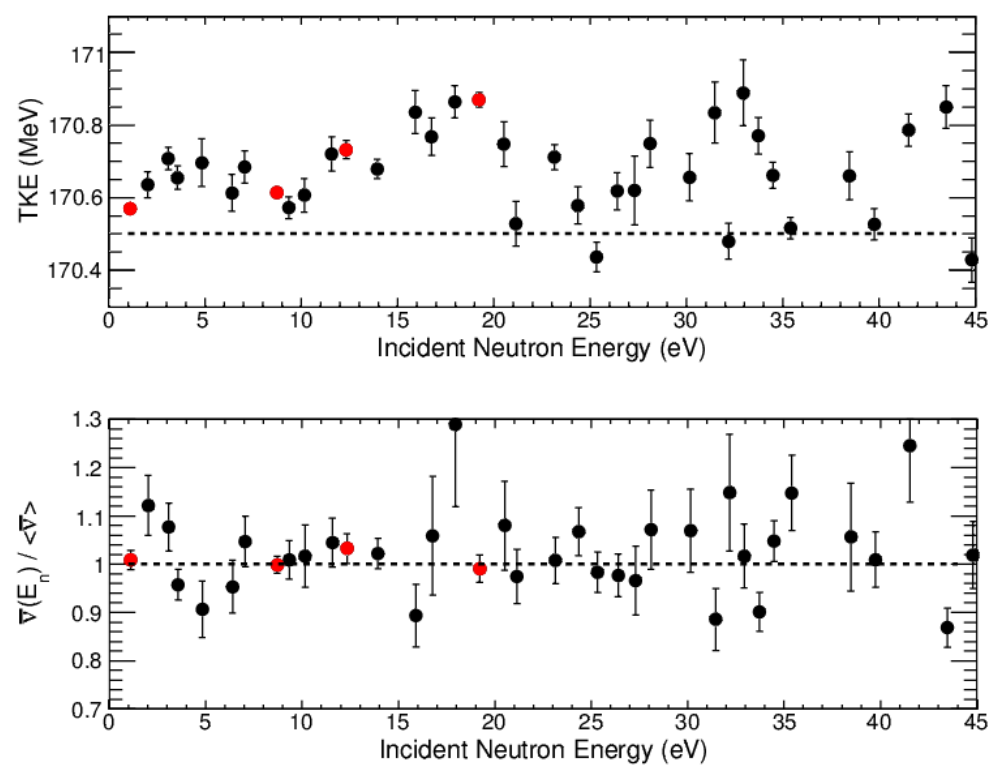

Figure 10. Upper part: Average TKE as a function of incident neutron energy for a given resonance or resonance groups in the energy range up to $45 \mathrm{eV}$, the dashed line refers to the thermal value. Lower part: Neutron multiplicity for the same resonances or resonance groups relative to the weighted average neutron multiplicity. The red points highlight strong resonances in the fission cross-section of ${ }^{235} \mathrm{U}$.

\subsection{Experimental results}

The spontaneous fission of ${ }^{252} \mathrm{Cf}$ was used to verify setup and analysis procedures relevant for studying neutron emission in resonance-neutron induced fission. Prompt fission neutron multiplicity correlations with fission fragment properties were investigated. Beside the purely experimental interest, recent advances in the theoretical description of prompt emission in fission (see e.g. Ref. [16]) require detailed input data for verifying the models. The experiment is essentially a reproduction of the experiment performed by Budtz-Jørgensen and Knitter [17]. Experimental details and results have been published elsewhere [14], a few selected results are repeated in the following. 
The mass dependence of the prompt fission neutron multiplicity per fragment from this experiment is compared to literature data in Fig. 8. The data from this study agree quite well with experimental data available in the literature and show in contrast to the data of Ref. [17] a bump at mass number 80 to 100. In Fig. 9 the mean number of neutrons emitted per fission is shown as a function of the TKE of the fission fragments. Except for low TKE the dependence is nearly linear. A least square fit to the data from the present experiment gives an inverse slope of dTKE/dv $=(12.6+/-$ $0.2) \mathrm{MeV} / \mathrm{n}$. Due to the correlation between TKE and fragment mass distributions this quantity must not be interpreted as the energy cost to emit one more neutron, as noted already by Nifenecker et al. [18]. A more detailed analysis of dTKE/dv for separated mass splits results in an average energy cost per neutron of $7.3 \mathrm{MeV} / \mathrm{n}$. Recently, discrepancies in the available experimental data on the behaviour of the total number of neutrons emitted as a function of total kinetic energy have been identified (see e.g. Ref. [19]), therefore efforts were made to identify experimental factors that may influence the result. All of the literature data included in Fig. 9, except one, were extracted using similar techniques as the present study. The factor having the strongest impact on the slope of $v_{\mathrm{T}}(\mathrm{TKE})$ is the correction for recoil energy imparted to the fission fragment by the detected neutron [20]. This was apparently not properly taken into account in the two deviating data sets in Fig. 9. A more detailed discussion of the results can be found in Ref. [14].
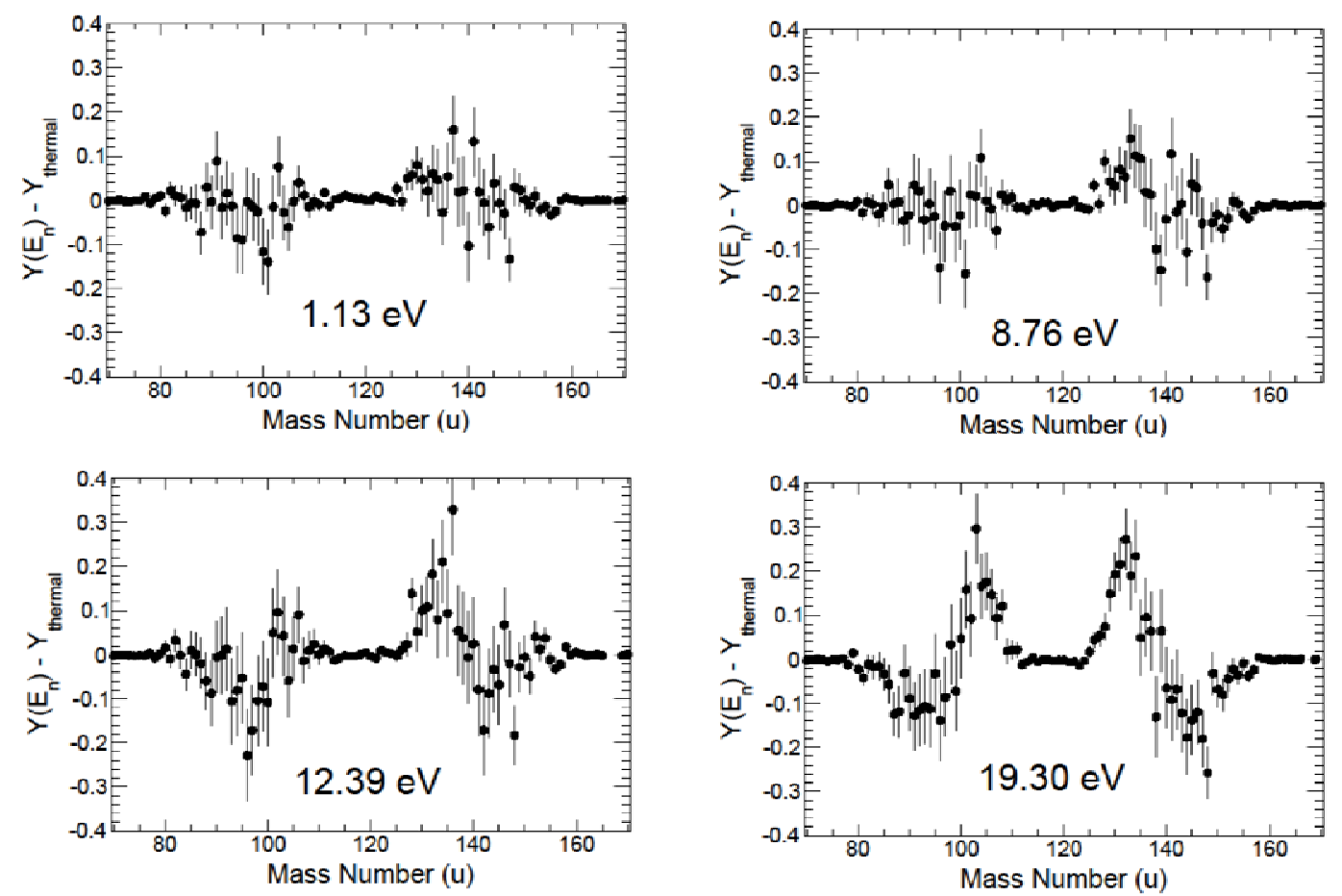

Figure 11. Changes in the mass yield distributions for selected strong resonances of which the energy is given in the inset. The yield differences are calculated to the mass yield distribution at thermal incident neutron energy.

For the prompt fission neutron multiplicity experiment on ${ }^{235} \mathrm{U}(\mathrm{n}, \mathrm{f})$, the SCINTIA array (Figs. 6,7) was employed to boost statistics. Preliminary results from a first experimental campaign in the first half of 2015 are shown. In the upper part of Fig. 10 the TKE of fission fragments is plotted as a function of the resonance energy. The TKE shows a strongly fluctuating behaviour as a function of the incident neutron energy, confirming the results of Ref. [9].

In some regions a change in incident neutron energy of only a few eV changes the TKE by more than $10^{5} \mathrm{eV}$. The lower part of Fig. 10 shows relative neutron multiplicities for the same resonance 
gates as in the upper part. At the present statistical accuracy no statistically significant fluctuations are found.

In Fig. 11 preliminary data on the changes in the fission fragment mass distribution for the strongest resonances are shown. At least the resonances at $12.39 \mathrm{eV}$ and $19.30 \mathrm{eV}$ show a clear change of the fission fragment yield in the inner and other wings of the asymmetric mass distributions. The change amounts to $\pm 0.3 \% / \mathrm{u}$ compared to the thermal neutron induced fission yield. Given that the thermal yield is in the order of $6 \% / \mathrm{u}$ this is a relative change of $\pm 5 \%$. Again this confirms previous results published in Ref. [9]. Further analysis is in progress.

\section{Prompt $\gamma$-ray emission in fission}

The fact that discrepancies have been observed in various benchmarks for the calculated total $\gamma$ heating relative to the measured one in the range from $10 \%$ to $28 \%$ [21], whereas an accuracy of $7.5 \%$ is requested, triggered increased interest in new measurements. These new experimental efforts were motivated by requests for new characteristic spectral data, especially for $\gamma$-ray multiplicity and mean photon energy per fission in thermal- and fast-neutron induced fission of ${ }^{235} \mathrm{U}$ and ${ }^{239} \mathrm{Pu}$ [22]. Both isotopes are considered the most important ones with respect to the modelling of innovative cores required for fast-spectrum Generation-IV reactors [23]. Instrumental advancements like the development of new $\gamma$-ray detectors as well as digital data-acquisition systems made the determination of new and improved prompt fission $\gamma$-ray spectral (PFGS) characteristics possible with high precision.

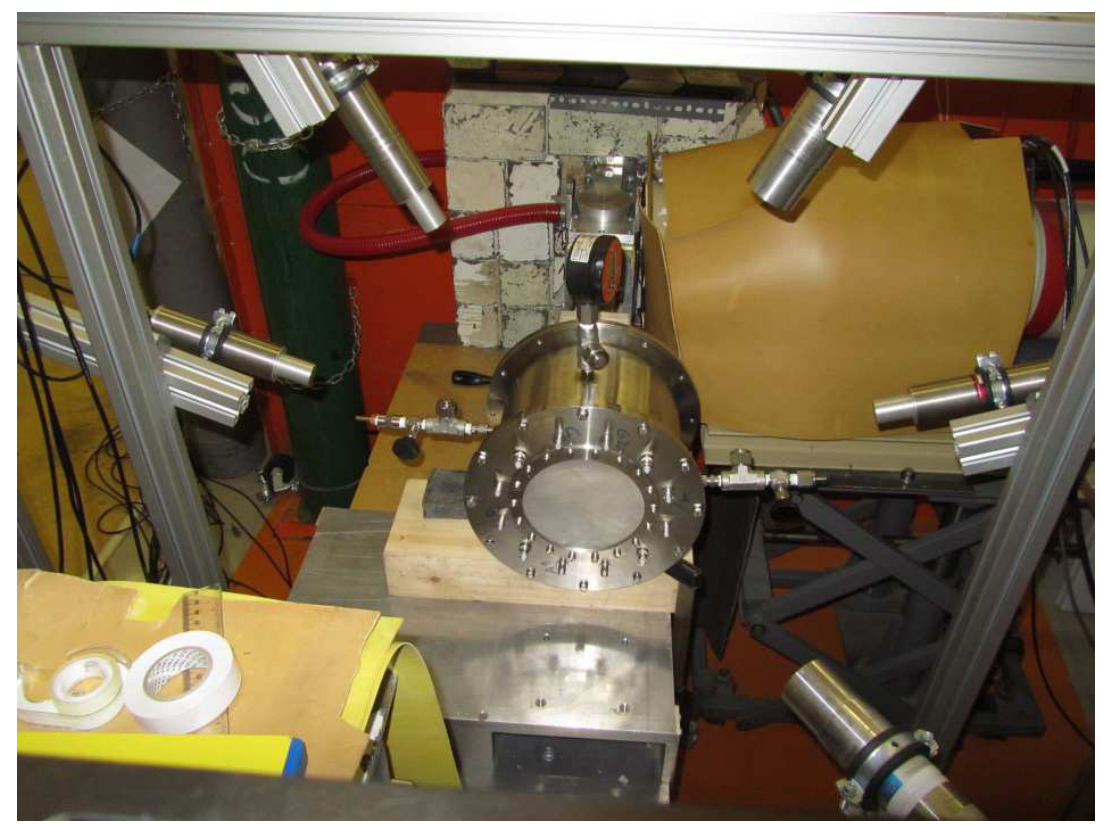

Figure 12. Typical set-up for measuring prompt fission $\gamma$-rays at the cold-neutron beam at the KFKI research reactor in Budapest. The fissile actinide sample is mounted in a Frisch-grid ionization chamber.

\subsection{Experimental setup}

To minimize the uncertainty in determining the $\gamma$-ray multiplicity and total released energy, three important detector characteristics need to be considered: (1) energy resolution, (2) intrinsic full peak efficiency and (3) timing resolution. Improved characteristics compared to previously used $\mathrm{NaI}$ 
detectors are nowadays found in cerium-doped lanthanum-halide $\left(\mathrm{LaBr}_{3}: \mathrm{Ce}\right)$ and cerium-halide scintillation detectors $\left(\mathrm{CeBr}_{3}\right)$. Such detectors have been used in the present experimental campaigns

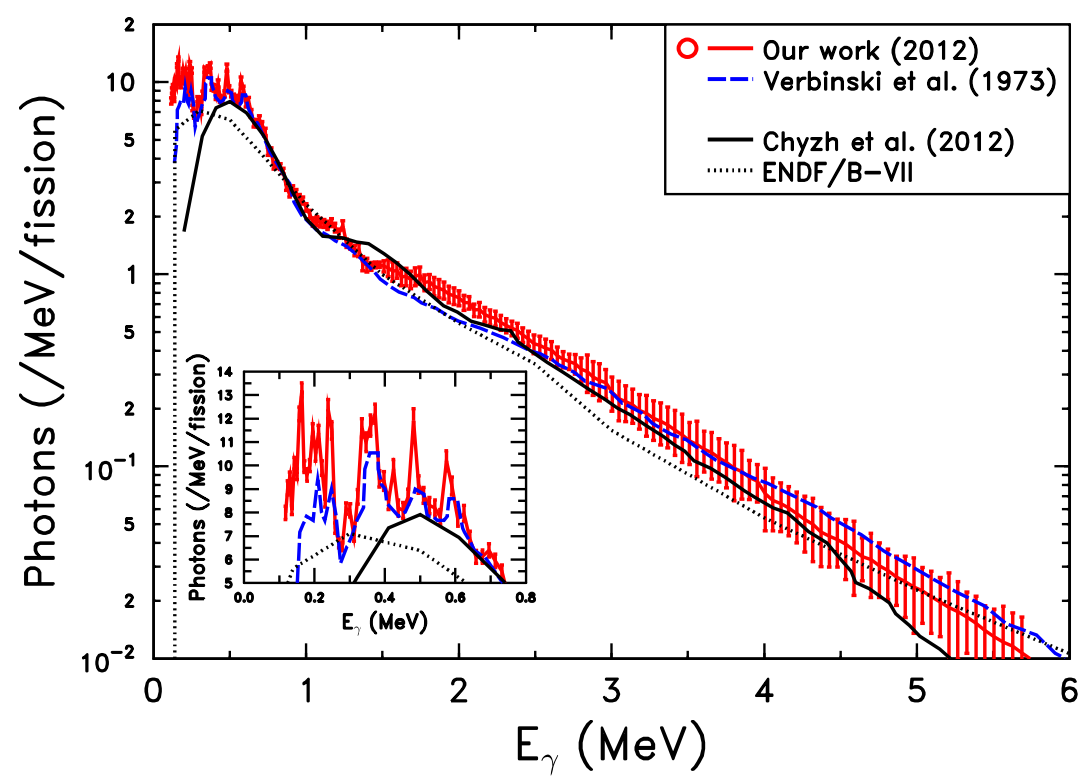

Figure 13. Prompt fission $\gamma$-ray spectrum from the spontaneous fission from 252Cf [24] compared to the historical data of Verbinski et al. [25] and recent measurements at LANL [26] (full black line).

together with a twin Frisch grid ionisation chamber as fission fragment detector giving also the trigger for the PFGS measurements. Measurements have been performed for ${ }^{252} \mathrm{Cf}(\mathrm{SF})$ and at thermal beam of the Budapest Research reactor for ${ }^{235} \mathrm{U}\left(\mathrm{n}_{\mathrm{th}}, \mathrm{f}\right.$ and ${ }^{241} \mathrm{Pu}\left(\mathrm{n}_{\mathrm{th}}, \mathrm{f}\right)$. A summary of the present status is given in Ref. [24]. A few highlights are given in the following.

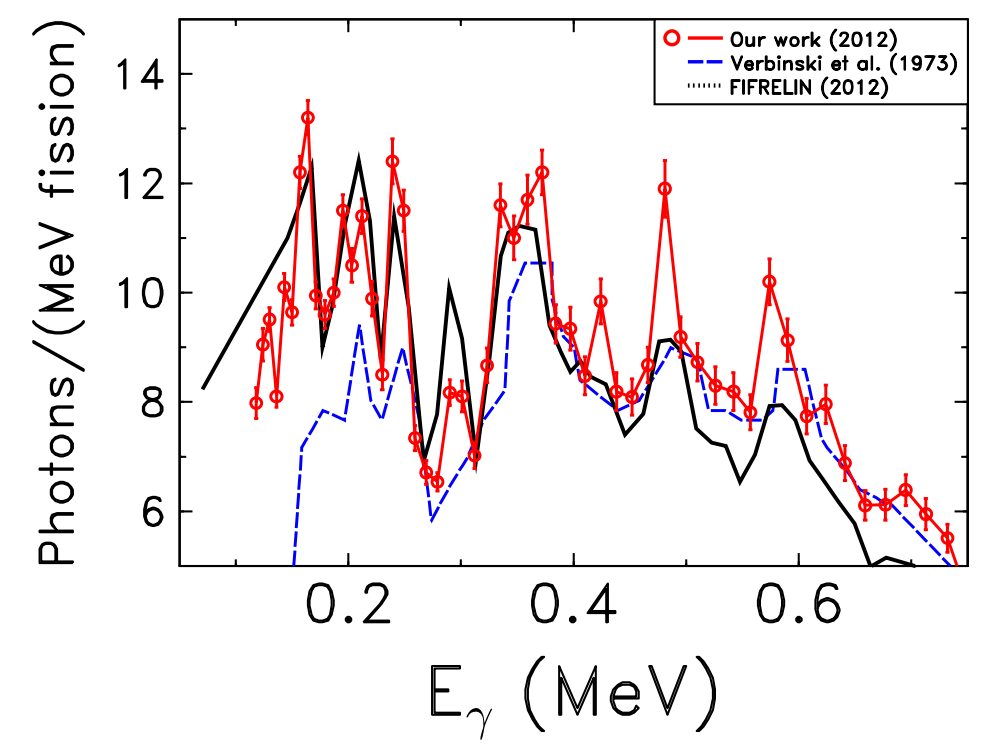

Figure 14. Prompt fission $\gamma$-ray spectrum from the spontaneous fission from $252 \mathrm{Cf}$ [24] compared to the historical data of Verbinski et al. [25] and recent FIFRELIN model calculations [27]. The dotted line represents the evaluated data ENDF/B-VII.1. 
In Fig. 13 an example of the PFGS of ${ }^{252} \mathrm{Cf}(\mathrm{sf})$ is given and compared to the historical measurements of Verbinski et al. [25] as well as to measurements using the DANCE detector at Los Alamos National Laboratory (LANL) [26] and the ENDF/B-VII evaluation. Compared to the literature data a more complex structure especially in the low $\gamma$-ray energy part is observed as given in the insert of Fig. 13.

In Fig. 14 a zoom of the low energy part of the PFGS is given. In addition to a comparison with Ref. [25] also a comparison with recent model calculations [27] is shown. The agreement is rather remarkable as the model calculation is able to reproduce well the observed structure which rules out the structureless and, hence, unphysical evaluation.

The low-energy structure observed in the PFGS from ${ }^{252} \mathrm{Cf}(\mathrm{sf})$ is also visible in the spectra from thermal-neutron induced fission on ${ }^{235} \mathrm{U}$ and ${ }^{241} \mathrm{Pu}$ with the individual peaks showing different intensities [24].

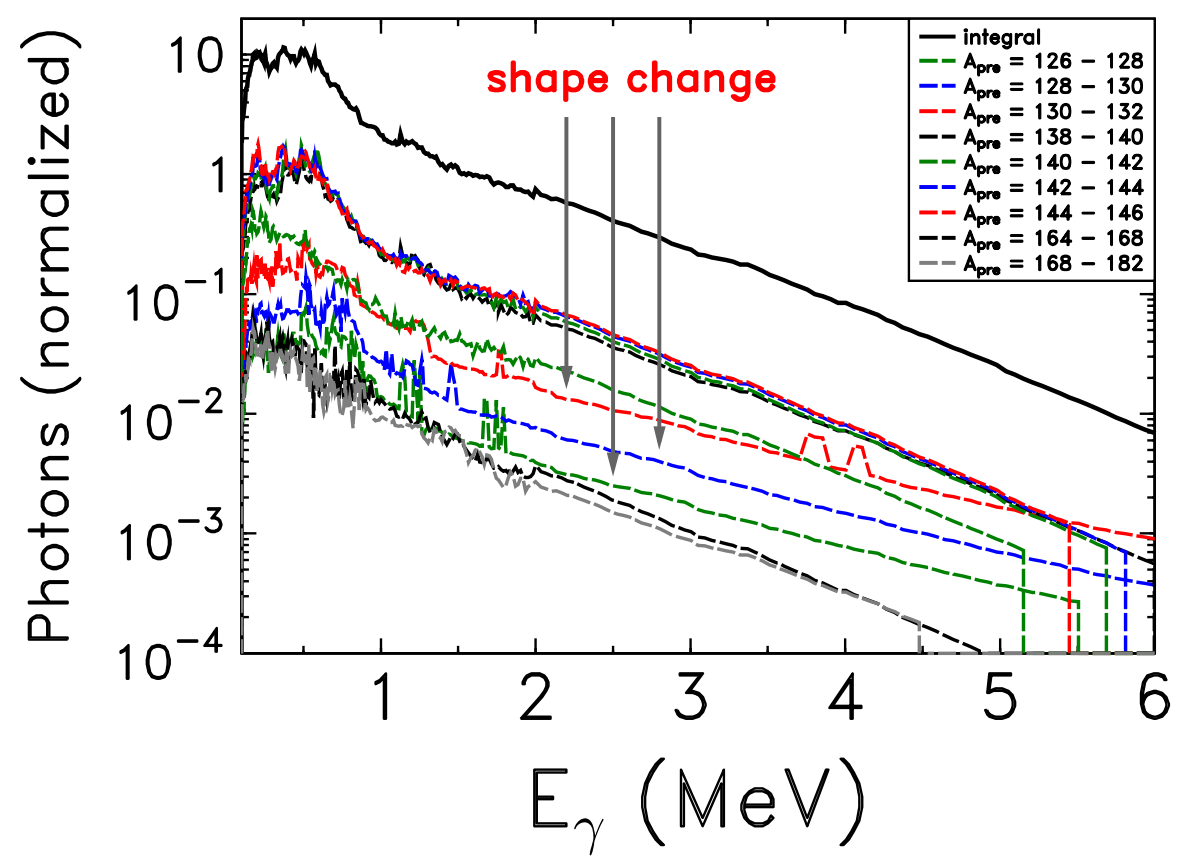

Figure 15. Prompt fission $\gamma$-ray spectra as a function of the heavy pre-neutron fission-fragment mass, $A_{\text {pre }}$, shown in logarithmic scale. Each mass cut contains also the contribution from the complementary light fragment, $A_{\text {comp }}$. Indicated is the significant spectral shape change around $\mathrm{E}=2.5 \mathrm{MeV}$ for masses between $\mathrm{A}_{\text {pre }}=126$ and $\mathrm{A}_{\text {pre }}=$ 132 (including $\mathrm{A}_{\text {comp }}=126$ down to $\mathrm{A}_{\text {comp }}=120$ ).

This may be due to the different shape of the corresponding mass distributions. To study this intriguing feature of the PFGS in more detail correlations of PFGS with fission-fragment properties have been investigated in the spontaneous fission of ${ }^{252} \mathrm{Cf}$. The focus was on a possible link between the (low-energy) structure and particular mass splits, and on whether the PFGS characteristics change significantly as a function of mass. The PFGS has been analysed as a function of the heavy fragment mass, in Fig. 15 shown for mass cuts of 2 mass units. Apart from the strongly changing structure at $\gamma$ ray energies below $1 \mathrm{MeV}$, a distinct shape change at mass splits with a heavy mass around or smaller than $\mathrm{A}=132$ is observed. A harder $\gamma$-ray spectrum was already observed in the 1990s [28] in an experiment with a $4 \pi \mathrm{NaI}$-detector array and attributed to non-statistical $\gamma$-emission with $\mathrm{E}>3.5 \mathrm{MeV}$ from spherical fission fragments. The present data shows that the shape change starts already around 
2.5 MeV. Of course, more data is necessary for a quantitative explanation in terms of fission fragment mass and total kinetic energy.

\section{Conclusions}

The present report gives an overview of the recent research highlights at JRC-IRMM. Progress has been made in recent years on the improvement of important fission cross-sections for innovative reactor system. With the help of digital data acquisition and digital signal processing together with arrays of detector systems for prompt neutron and $\gamma$-ray emission more precise measurement capabilities have opened up. For ${ }^{252} \mathrm{Cf}(\mathrm{sf})$ the question on the slope of the neutron multiplicity as a function of TKE has been solved. No strong fluctuations in the prompt neutron multiplicity for ${ }^{235} \mathrm{U}(\mathrm{n}, \mathrm{f})$ in resonance induced fission has been found, but fluctuations in mass yield and TKE distributions already mentioned in Ref. [9] have been confirmed. More results concerning this experiment are to be expected. Concerning prompt $\gamma$-ray emission in fission, improved detector systems have confirmed, with better energy resolution, the structure at low $\gamma$-ray energies observed already in Ref. [25]. In addition a shape change has been found for specific masses around the double magic mass $A=132$ confirming results from Ref. [28]. More experiments are planned both at thermal and higher incident neutron energies.

\section{Acknowledgements}

Three of the authors (P. S-C, R. B. and A. G.) are indebted to the European Commission for providing a fellowship at EC-JRC IRMM, during which part of this work was carried out. This work was also supported by the European Commission in the frame works of the FP7-ERINDA (agreement number 269499), ANDES (agreement number 249671) and CHANDA (agreement number 605203) programme, which is hereby gratefully acknowledged. The authors are very grateful to the colleagues at the KFKI Budapest for their invaluable support during several measurement campaigns.

\section{References}

1. M. Salvatores, Uncertainty and Target Accuracy Assessment for Innovative Systems Using Recent Covariance Data Evaluations (NEA/WPEC-26) (Nuclear Energy Agency (OECD), 2008).

2. C. Lubitz, R. Roussin, NEA/WPEC 18 (1999)

3. C. De Saint Jean, R. McKnight, NEA/WPEC 34 (2014)

4. NEA Nuclear Data High Priority Request List https://www.oecd-nea.org/dbdata/hprl/

5. P. Salvador-Castiñeira, T. Brys, R. Eykens, F.-J. Hambsch, A. Göök, A. Moens, S. Oberstedt, G. Sibbens, D. Vanleeuw, M. Vidali, and C. Pretel, Phys. Rev. C 92, 014620 (2015)

6. P. Salvador-Castiñeira, T. Brys, R. Eykens, F.-J. Hambsch, A. Göök, A. Moens, S. Oberstedt, G. Sibbens, D. Vanleeuw, M. Vidali, and C. Pretel, Phys. Rev. C 92, 044606 (2015)

7. P. Salvador-Castiñeira, PhD thesis, University Polytecnica de Catalunya, Barcelona (2014).

8. P. Salvador-Castiñeira, T. Brys, R. Eykens, F.-J. Hambsch, A. Moens, S. Oberstedt, G. Sibbens, D. Vanleeuw, M. Vidali, and C. Pretel, Phys. Rev. C 88, 064611 (2013)

9. F.-J. Hambsch, H.-H. Knitter, C. Budtz-Jørgensen, J.P. Theobald, Nucl. Phys. A491, 56.(1989)

10. F.-J. Hambsch, I. Ruskov, L. Dematte, in: Proc. Scientific Workshop on Nuclear Fission Dynamics and the Emission of Prompt Neutrons and Gamma Rays (Theory-1), (2011) EUR 24802, Sinaia, Romania. p. 41.

11. R.E. Howe, T.W. Phillips, C.D. Bowman, Phys. Rev. C 13, 195 (1976)

12. R. Gwin, R.R. Spencer, R.W. Ingle, Nucl. Sci. Eng. 87, 381 (1984)

13. A. Al-Adili, F.-J. Hambsch, S. Pomp, S. Oberstedt, Phys. Rev. C 86, 054601 (2012)

14. A. Göök, F.-J. Hambsch, M. Vidali, Phys. Rev. C 90, 064611 (2014)

15. A. Göök, F.-J. Hambsch, M. Vidali, Phys. Procedia. 64, 190 (2015) 
16. O. Litaize, O. Serot, Phys. Rev. C 82, 054616 (2010)

17. C. Budtz-Jørgensen, H.-H. Knitter, Nucl. Phys. A490, 307 (1988)

18. H. Nifenecker, C. Signarbieux, R. Babinet, J. Poitou, in: Proc. 3rd Symp. on Physics and Chemistry of Fission, IAEA, Vienna. (1974) p. 51

19. A. Gavron, Nucl. Inst. and Meth. 115, 99 (1974)

20. A. Tudora, Ann. Nucl. Energy 53, 507 (2013)

21. A. Lüthi, R. Chawla, and G. Rimpault, Nucl. Sci. Eng. 138, 3 (2001)

22. Nuclear Data High Priority Request List of the NEA (Req. ID:H.3, H.4), http://www.nea.fr/html/dbdata/hprl/hprlview.pl?ID=421,

http://www.nea.fr/html/dbdata/hprl/hprlview.pl?ID=422

23. G. Rimpault, D. Bernard, D. Blanchet, C. Vaglio-Gaudard, S. Ravaux, and A. Santamaria, Phys. Procedia 31, 3 (2012)

24. S. Oberstedt, R. Billnert, A. Gatera, W. Geerts, P. Halipré, F.-J. Hambsch, M. Lebois, A. Oberstedt, P. Marini, M. Vidali, J.N. Wilson, Phys. Procedia. 64, 83 (2015)

25. V.V. Verbinski, H. Weber, and R.E. Sund, Phys. Rev C 7, 1173 (1973)

26. A. Chyzh, C.Y. Wu, E. Kwan, R.A. Henderson, J.M. Gostic, T.A. Bredeweg, R.C. Haight, A.C. Hayes-Sterbenz, M. Jandel, J.M. ODonnell, J.L. Ullmann, Phys. Rev. C 85, 021601 (2012)

27. D. Regnier, private communication (2013)

28. A. Hotzel, P. Thirolf, Ch. Ender, D. Schwalm, M. Mutterer, P. Singer, M. Klemens, J.P. Theobald, M. Hesse., F. Gönnenwein, H. van den Ploeg, Z. Physik A 356, 299 (1996). 